Revue française de la traduction

\title{
Aspects lexico-sémantiques de la traduction technique du français vers le roumain
}

Elena-Cristina Ilinca et Ana-Marina Tomescu

\section{(2) OpenEdition}

Journals

Édition électronique

URL : http://journals.openedition.org/traduire/534

DOI : $10.4000 /$ traduire.534

ISSN : 2272-9992

Éditeur

Société française des traducteurs

Édition imprimée

Date de publication : 1 juin 2013

Pagination : 68-80

ISSN : 0395-773X

\section{Référence électronique}

Elena-Cristina Ilinca et Ana-Marina Tomescu, « Aspects lexico-sémantiques de la traduction technique du français vers le roumain », Traduire [En ligne], 228 | 2013, mis en ligne le 01 juin 2015, consulté le 19 avril 2019. URL : http://journals.openedition.org/traduire/534 ; DOI : 10.4000/traduire.534 


\section{Aspects lexico-sémantiques de la traduction technique du français vers le roumain}

Elena-Cristina Ilinca - Ana-Marina Tomescu

Ayant comme objectif la transmission d'informations à des destinataires plus ou moins définis, la traduction spécialisée est un élément important dans la communication d'informations technoscientifiques dans notre société.

Selon Vande Walle (2007, apud Scarpa, 2010 : 95), en 2006, $99 \%$ des textes traduits en Europe étaient des textes dans les domaines technique (39\%), commercial (26\%), juridique (12\%), médical ( $9 \%$ ), administratif $(9 \%)$ et scientifique (4\%). $1 \%$ des textes traduits étaient des textes à caractère général. Nous pouvons donc remarquer la prédominance de la traduction technique par rapport aux autres types de traduction spécialisée.

Dans de nombreux ouvrages traitant de la traduction spécialisée, les domaines de la science et de la technique sont pris comme un ensemble. Or, il existe des approches théoriques qui font la distinction entre le langage scientifique et le langage technique. En retenant cette distinction, notre étude se propose d'aborder quelques aspects de la traduction technique du français au roumain. Après avoir défini la traduction spécialisée et présenté ses principales caractéristiques, nous la mettrons en parallèle avec la traduction littéraire. Ensuite, nous identifierons et discuterons des difficultés que la traduction technique du français au roumain peut engendrer au niveau lexico-sémantique et proposerons des solutions de traduction en analysant les spécificités et la potentialité des deux systèmes linguistiques.

\section{Discours scientifique versus discours technique}

Selon des critères sémiotiques et discursifs, le discours scientifique repose sur des concepts épistémologiques ayant comme rôle principal la transmission d'une connaissance scientifique, alors que le discours technique a une finalité pratique plutôt liée à un savoir-faire, (Vigner \& Martin, 1976). La distinction entre lexique technique et lexique scientifique tient donc au " degré de spécificité " des termes des deux lexiques: 
Le lexique technique c'est le lexique propre aux spécialités considérées en elles-mêmes, surtout au stade des manipulations et de l'application pratique [...], le lexique scientifique général est commun à toutes les spécialités. [...] Les termes du lexique scientifique général sont porteurs d'une information plus largement utilisable mais moins complète et moins précise que celle qui est véhiculée par les termes du lexique technique. (Cuniţă)(1),

Louis Guilbert(2) précise lui aussi les particularités des vocabulaires scientifique et technique :

Terme scientifique : milieu homogène de spécialistes de même culture, de même formation, [...] communication à un haut niveau d'élaboration conceptuelle et de rigueur dans l'analyse, [...] énonciation écrite, [...] monovalence [...]. Les termes employés ne s'étendent pas à la communauté linguistique tout entière, sauf si tel concept vient à jouir d'une extension considérable, auquel cas il cesse d'être proprement un terme scientifique. Les techniciens représentent une catégorie de locuteurs non homogène, [...] cadres et ouvriers. Des niveaux de langue peuvent traverser les vocabulaires techniques, [...] échange généralisé entre les termes proprement " techniques " et le lexique général.

Marie-Françoise Mortureux(3) affirme à cet égard :

Le rôle d'un terme scientifique est de nommer un concept destiné à rendre compte d'une façon unifiée et abstraite de phénomènes divers, disparates en apparence, parfois contradictoires, dont la cooccurrence est appréhendée comme complémentaire; ainsi l'inflation rend-elle compte de la simultanéité de la hausse (des prix, des salaires, des taux d'intérêt...) et de la dépréciation de la monnaie (" plus on gagne, moins on est riche "). Un terme technique est plutôt le nom d'une matière première, d'un processus, d'un agent ou d'un instrument; les variations se fonderaient plutôt sur les métonymies reflétant en langue les relations qui existent entre ces différents référents.

Les critères de distinction présentés ci-dessus concernent les vocabulaires de la science et de la technique. Dans les grandes lignes, le terme scientifique renvoie à des réalités abstraites (concepts, phénomènes) alors que le terme technique désigne plutôt des réalités concrètes (applications et manipulations pratiques). Le critère de l'abstraction semble cependant difficile à manier : " la distinction entre abstrait et concret n'est plus évidente " (Beaune)(4). La frontière entre les domaines scientifique et technique est en effet très fragile, les deux activités s'entrecroisant sans cesse.
(1) $1980: 32$
(2) $1973: 15$
(3) $1995: 21$
(4) $1988: 72$. 


\section{Traduction spécialisée versus traduction littéraire}

La traduction spécialisée est généralement définie par rapport à la traduction littéraire. La traduction littéraire a comme objectif de traduire la poésie, des récits, des romans, alors que la traduction spécialisée s'occupe de textes scientifiques, techniques, juridiques, commerciaux, etc. Il est impossible néanmoins de faire une distinction nette entre les deux catégories de traduction. Même si le traducteur utilise les mêmes procédés dans la traduction littéraire et spécialisée, il doit prendre en compte certaines caractéristiques textuelles : par exemple, l'individualité de l'auteur du texte ou la nature créative de ce dernier sont considérées comme des caractéristiques de la traduction littéraire (Scarpa)(5) alors que la traduction spécialisée a pour caractéristique d'être orientée vers son public de destination (Bell, 1995).

Une autre distinction est opérée par Rega(6), pour qui, dans la traduction littéraire, le texte représente un fait unique alors que, dans la traduction spécialisée, le traducteur peut faire appel à d'autres textes similaires dans la langue d'arrivée pour traduire son texte. L'étape de compréhension d'un texte est très importante pour tout type de traduction. Le niveau de langue et la tonalité du texte de départ sont deux éléments à prendre en compte dès cette étape. Le plus souvent, la composante graphique du texte est plus importante dans la traduction technique que dans la traduction littéraire. Hormis l'unité de sens formé par le paragraphe qui est commune aux deux types de traductions, dans la traduction technique le traducteur se confronte à des tableaux, schémas, figures, diagrammes, etc. Ce sont des images standardisées qui respectent des règles précises. Ces images constituent le " lieu commun " des producteurs et des utilisateurs de textes techniques (Froeliger)(7). Daniel Jacobi affirme à cet égard :

La place des illustrations, et plus généralement, de tous les signes non linguistiques du paratexte, l'organisation de l'aire scripturale visant à provoquer des lectures partielles, tout indique que dans le document scientifique la dimension scriptovisuelle tient une place majeure... Analyser l'usage social du discours scientifique suppose bien de parvenir à prendre en compte aussi sa dimension scriptovisuelle(8).

Un autre aspect important dans le cas de la traduction spécialisée constitue la recherche du terme approprié. Le problème de la documentation est dû au fait que les vocabulaires spécialisés sont toujours en expansion, cela grâce aux progrès enregistrés dans les domaines technique et scientifique. À ce niveau apparaissent des difficultés qui tiennent à l'instabilité sémantique des termes, à la néologie, à la polysémie, à la synonymie, aux emprunts ou aux calques. Selon Daniel Gile(9), les faiblesses dans la terminologie et la phraséologie spécialisée
(5) $2010: 95$.
(6) $2001: 52-69$.
(7) $2003: 41$
(8) $1984: 44$
(9) $2004: 217-218$. 
sont dues principalement à une recherche inappropriée, à un mauvais choix des sources ou à une mauvaise utilisation de celles-ci. A contrario, la traduction littéraire est plus souple, le choix d'un terme inapproprié n'ayant pas de conséquences graves sur la finalité du texte. Si dans le cas du texte littéraire les conséquences concrètes d'une décision en faveur de tel ou tel mot n'ont pas d'impact direct sur l'existence physique du lecteur présumé, dans le cas des textes scientifiques la responsabilité du traducteur a une toute autre portée, qui implique des conséquences parfois désastreuses dans l'activité du domaine en question.

L'étape de réexpression présuppose la mise en pratique du vocabulaire et de la langue. Le traducteur essaie de rendre dans le texte d'arrivée ce qu'il a compris du texte de départ. Pour la traduction littéraire, le texte traduit n'est pas seulement un moyen de communiquer des informations, le style de l'auteur du texte source étant un élément à préserver dans le texte cible. Dans la traduction spécialisée, le critère de la fidélité par rapport à la forme du texte de départ n'est pas nécessairement respecté, le traducteur s'attachant à rendre le contenu informatif du texte à traduire tout en respectant les normes et les conventions de la rédaction spécialisée dans la langue d'arrivée. Selon N. Froeliger(10), une bonne traduction technique doit réunir quatre qualités fondamentales :

- l'exhaustivité : le texte d'arrivée doit contenir toute l'information présente dans le texte de départ ;

- la monosémie : le texte d'arrivée doit avoir un sens et un seul ;

- la précision : un texte technique doit contenir un lexique précis, capable de transmettre un savoir donné ou des procédés d'application d'une connaissance à des fins pratiques ;

- l'accessibilité à l'ensemble de la collectivité visée : le texte d'arrivée doit être présenté dans la forme standard de la catégorie professionnelle du demandeur.

La révision de la traduction technique doit donc porter plus sur le fond que sur la forme. L'originalité du style d'une traduction technique est un aspect qui compte moins. Le degré de reproduction de l'information contenue dans le texte à traduire et le degré d'appropriation vis-à-vis des destinataires guideront la révision d'une traduction. L'appel à l'expert est une étape obligatoire de la révision de la traduction technique. Dans la traduction littéraire, l'originalité et la créativité du traducteur seront toujours des critères d'évaluation importants.

\section{Quelques difficultés de la traduction en roumain de termes techniques français}

Nous ne pouvons pas traiter la question de la traduction technique du français au roumain sans parler de l'influence exercée en général par la culture et la civilisation françaises sur la

(10) $2003: 37-39$. 
culture et la civilisation roumaines au long des siècles. Dès la moitié du Xville siècle cette influence est ressentie dans les milieux aristocratiques roumains qui font du français leur langue de communication. L'État français a, lui aussi, largement contribué à la diffusion du français sur le territoire des principautés roumaines. À cette époque-là le turc jouait encore un rôle prédominant dans le développement des langues balkaniques. De nombreux officiels français sont envoyés sur le territoire roumain, des services consulaires français sont instaurés afin de promouvoir la langue et la culture françaises. En 1859, la France reconnaît l'Union des Principautés Roumaines. Dès la fin du xixe siècle le français est enseigné par des professeurs français dans les écoles roumaines. Ce ne sont là que quelques faits ayant contribué à l'emprunt des pratiques françaises dans l'administration publique, dans la société, dans les mentalités roumaines. La langue roumaine subit, elle aussi, une forte influence française. Les xixe et xxe siècles connaissent une activité intense dans le domaine de la traduction du français au roumain : sont traduites des œuvres littéraires, des textes scientifiques, des textes techniques.

Le lexique roumain s'enrichit considérablement grâce aux emprunts néologiques du français qui devient ainsi la première source, suivi du latin, du grec moderne, de l'italien et de l'allemand (Coteanu \& Sala)(11). Les domaines les plus concernés sont les domaines technique et scientifique pour lesquels le lexique roumain était pauvre. Selon une étude effectuée par Dimitrie Macrea (1970) sur trois dictionnaires roumains(12), le lexique scientifique et technique roumain contient 62 à $95 \%$ d'éléments empruntés (directement ou indirectement) au latin, l'emprunt étant fait le plus souvent par l'intermédiaire du français. La plupart du temps, le transfert d'une langue à l'autre est accompagné d'une adaptation lexico-phonétique et graphique au roumain. Prenons quelques exemples du domaine de la technique :

phare/far ; moteur/motor ; suspension/suspensie ; adapteur/ adaptor ; marche arrière/ marşarier ; berline/berlină ; boîte de vitesse/ cutie de viteză ; transmission/ transmisie ; pédale/pedală ; volant/volan ; indicateur de vitesse /indicator de viteză ; ceinture de sécurité/ centură de securitate, par-brise/parbriz.

Lerat(13) nous rappelle que le rôle des néologismes est d'enrichir le vocabulaire pour des raisons de dénomination, d'expression ou de communication. Les néologismes sont importants à double titre.

Premièrement, ils servent à combler un vide terminologique dans la langue d'arrivée. C'est le cas des néologismes dénotatifs ou techniques, dont surtout les néonymes, les néologismes utilisés dans les langues de spécialité. Dans le domaine de la terminologie, Bălan-Mihailovici(14)

(11) $1987: 149$.

(12) (Le dictionnaire technique polyglotte, 1963, Le dictionnaire encyclopédique roumain, 1962-1966, Le dictionnaire de la langue roumaine moderne, 1958)

(13) $1993: 132$.

(14) $2005: 23-29$. 
distingue deux types de néologies : la néologie primaire, qui répond à une nécessité immédiate, donc à valeur dénotative et la néologie traductive, lorsque de nouveaux termes apparaissent dans la langue source et le traducteur doit trouver un équivalent dans la langue cible. C'est surtout dans le domaine de la technique et de la science que la découverte de nouveaux concepts et la création de nouveaux dispositifs ou objets mènent à la création de noms qui correspondent à ces nouvelles réalités. Le plus souvent c'est au traducteur de combler ces lacunes lexicales. Prenons quelques exemples de termes anglais du domaine de l'informatique utilisés en roumain : log-out, tagline, cookie, etc. En général les emprunts lexicaux venus de l'anglais ces dernières décennies ne sont pas adaptés au roumain du point de vue lexico-phonétique et graphique. Ce phénomène s'explique par le fait que l'anglais est devenu la langue la plus utilisée dans les milieux professionnels roumains et par le fait que la technique et la science progressent très rapidement, la langue ayant besoin de plus de temps pour assimiler et adapter les termes renvoyant à des nouvelles réalités.

Deuxièmement, les néologismes servent à nuancer le vocabulaire d'une langue. Cette catégorie de néologismes stylistiques ou connotatifs comprend des mots néologiques qui ont des synonymes dans la langue cible, le rôle étant de nuancer le vocabulaire. Par exemple, dans la langue générale roumaine il y a la série synonymique deosebit- diferit- divers-felurit- variat, formée de néologismes provenant du français (varié, différent, divers) et des termes déjà existants en roumain (felurit, deosebit). Ce fait est rare en langage technique, les termes techniques devant être très précis.

Même si le lexique technique roumain contient beaucoup de termes empruntés au français, le vocabulaire constitue le niveau le plus problématique de la traduction technique du français au roumain. Nous allons maintenant nous arrêter sur les procédés les plus fréquents de formation du lexique technique français qui peuvent constituer des sources d'ambiguïté sémantique voire même de fautes de sens dans la traduction.

Les procédés de la dérivation et de la composition jouent un rôle très important dans la dynamique de la terminologie technique française. Tout d'abord prenons le cas du procédé de la dérivation suffixale. Les suffixes français les plus utilisés et les plus productifs dans la dérivation des termes techniques sont -age, -ment et -ion (avec leurs variantes : -ement, -tion, -ssion, -xion, -ation, -ition, -ification, -isation).

Voici quelques exemples de termes français dérivés qui sont passés en roumain par les procédés de l'emprunt ou du calque :

accorder/a acorda, accord/acord, accordage/acordaj ;

adapter/a adapta, adaptateur/adaptator, adaptatif/adaptativ, adaption/adaptare ; goudronner/gudrona, goudron/gudron, goudronnage/ gudronaj, gudronare, goudronneuse/ gudroneză ;

granule/granulă, granulation/granulare, granulaţie, granulaire/granular, granularité/granularitate, granulateur/granulator. 
En analysant les exemples ci-dessus, nous pouvons constater que le découpage de la réalité peut être plus analytique dans une langue que dans l'autre. II existe des cas où pour exprimer l'action et le résultat de l'action, le français utilise un seul mot alors que le roumain se sert de deux, voire trois mots dérivés différents. Ce sont des cas de focalisation : « Il y a généralisation dans une langue $L$ quand on emploie un lexème dont l'extension est plus grande que celle de son hétéronyme en L', et particularisation lorsque, pour le même lexème, on se sert de deux ou de plusieurs hétéronymes. Dans le premier cas on parle de focalisation et dans le second de scission. " (Cristea)(15). Prenons trois exemples qui peuvent poser problème en situation de traduction :

- injection - injectare (l'action d'injecter), injecţie (résultat de l'action d'injecter) ;

- compression - comprimare, compresiune (l'action de comprimer), compresie (résultat de l'action de comprimer) ;

- composition - compunere (l'action de composer), compoziţie (le résultat de l'action de composer).

Dans une phrase du type : "L'injection du gazole commence en fin de compression ", le terme " injection " sera rendu par injectare car il désigne le processus d'injection. De même, le terme " compression " désigne l'action de comprimer et il sera traduit par comprimare. Par contre, dans une phrase comme "Tous les moteurs à essence peuvent recevoir un système d'injection directe... ", le terme " injection " désigne le résultat de l'action et il sera traduit par injecţie.

Dans un texte sur l'industrie de l'automobile contenant le terme " compression à froid ", l'équivalent roumain approprié serait compresie la rece alors que dans un texte sur les techniques d'obtention de l'huile d'olive, le traducteur choisira comme équivalent comprimare la rece. Dans un texte portant sur la mécanique, " compression adiabatique " aura comme équivalents en roumain soit compresiune adiabatică soit comprimare adiabatică.

Dans un texte portant sur l'industrie nucléaire, " composition isotopique " sera rendu en roumain par compoziţie izotopică alors que dans un texte sur la mécanique, " composition de mouvements " trouvera son équivalent en compunere de mişcări. En roumain il existe aussi le terme compoziţie de mişcări mais c'est un terme spécifique au domaine de la danse. C'est donc le domaine et le contexte linguistique qui régissent le choix de l'équivalent adéquat.

La traduction des mots polysémiques est un autre aspect problématique de la traduction technique du français au roumain. Prenons le cas du mot " tranche " dont le sens varie selon le domaine et qui pourrait poser problème lors de sa traduction du français ou roumain. Le dictionnaire Larousse fournit les sens suivants :

(15) $2007: 51$. 
Agriculture : terre que la charrue soulève et déverse sur le côté en traçant le sillon (équivalent roumain brazdă).

Boucherie : synonyme de " tende de tranche " (morceau de la cuisse du bœuf, débité en rôtis) (équivalent roumain felie).

Carrières : ligne de saignée dans un banc de pierres ou bloc mince de pierre ou de marbre (équivalents roumains fâşie, felie).

Chemin de fer : groupe de wagons ou de voiture entrant dans la composition d'un train et ayant la même destination (équivalent roumain tranşăa).

Droit : chacune des différentes strates du revenu des personnes physiques, soumis à des taux d'imposition différents (équivalent roumain tranşă).

Électricité : unité de production d'énergie électrique (équivalent roumain unitate de producţie).

Mathématiques : ensemble de chiffres consécutifs dans l'écriture d'un nombre (équivalent roumain tranşă).

Météorologie : couche d'eau liquide précipitée sur le sol dont on mesure l'épaisseur à l'aide d'un pluviomètre (équivalent roumain fâşie).

Mines : portion de gisement constituant une unité d'exploitation intermédiaire entre la volée et le panneau (équivalent roumain fâşie).

Monnaies : surface que présente, sur son pourtour, l'épaisseur d'une pièce de monnaie (équivalent roumain muchie).

Reliure : chacune des trois surfaces visibles correspondant à l'épaisseur des cahiers d'un livre, entre les plats (équivalent roumain tranşă).

Télécommunications : subdivision d'un programme de radio ou de télévision (équivalent roumain divizie).

Nous pouvons remarquer que le mot " tranche " a plusieurs équivalents en roumain : felie, tranşă, fâşie, divizie, muchie, brazdă, unitate de producție. Dans un texte sur les centrales nucléaires, le terme " tranche " signifiant " unité de production d'énergie électrique " pourrait constituer un obstacle : "Toutes les fonctions importantes d'une tranche nucléaire sont commandées et surveillées depuis la salle de commande... ". Dans ce sens, beaucoup de chercheurs ont accordé une importance spéciale à la lexicologie contrastive. Ils ont élaboré des dictionnaires scientifico-techniques polyglottes. Ils attirent l'attention sur le fait qu'un terme monosémique dans une langue devient polysémique par confrontation à une autre, d'où la conclusion qu'au " niveau de la terminologie, la monosémie absolue s'avère un mythe " (Gentilhomme)(16).

(16) $1984: 32$. 
Si un traducteur devait traduire le terme du français en roumain, il aurait du mal à trouver un bon équivalent sans une documentation sérieuse au préalable. Choisir comme équivalent unitate de producţie serait préférable à sector ou divizie. Le terme unitate de producţie constitue plutôt la traduction littérale de l'explication du dictionnaire du terme " tranche " (unité de production) mais c'est le terme consacré en roumain pour désigner l'ensemble constitué du réacteur et du système de production d'électricité d'une centrale nucléaire.

À la lumière de ce que nous venons de voir, nous pouvons dire que, dans le cas d'une différence d'extension entre les hétéronymes, la difficulté est double : il faut d'une part identifier les traits sémantiques qui sont lexémisés, et d'autre part circonscrire les restrictions sélectives propres aux hétéronymes.

La composition nominale est fortement utilisée au profit de la dénomination des concepts techniques ou scientifiques:

Cette fonction de dénomination fait que le composé ne peut être validé comme dénomination que par un professionnel, même si sa reconnaissance et son analyse sont facilitées par des tests linguistiques et le repérage des candidats rendu possible par un simple calcul statistique portant sur les cooccurrences lexicales. II faut bien voir en effet qu'un composé relevant du vocabulaire technique n'a pas de spécificité grammaticale par rapport à un composé non technique mais que sa structure dépend entièrement des propriétés typologiques générales de telle langue (Lerat)(17).

Dans le cas du procédé de la composition, le procédé le plus fréquent à la base du lexique technique est le prolongement du sens d'une unité lexicale appartenant à la langue générale à une utilisation spécifique dans un domaine spécialisé. Les termes du lexique technique sont ainsi très souvent empruntés à des mots du lexique général ; c'est ce qu'on appelle le passage du mot au terme(18).

Exemples :

Arbre : arbre à cames (construction automobile), arbre de distribution (construction automobile), arbre de défaillances (technologie), arbre de roue (construction d'automobiles), arbre de transmission (construction automobile), arbre de boite de vitesse (construction automobile), arbre de pompe (technologie), arbre à cardan (construction automobile), arbre vilebrequin (construction automobile), arbre articulé (construction automobile), etc.

Champ : champ accélérateur (électrotechnique, mécanique, technologie nucléaire), champ acoustique (acoustique), champ à haute fréquence (électrotechnique, télécommunications),

(17) $1997: 12$.

(18) Voir à cet égard PETIT, Gérard, 2001, "L'introuvable identité du terme technique ", Revue française de linguistique appliquée $\mathrm{VI}, 2$, (2001), p. 63-79. 
champ alternatif (électrotechnique), champ antagoniste (électrotechnique, nucléaire), champ apparent (optique), champ d'aimantation (magnétisme), champ de déflexion (électronique), champ de gravitation (mécanique), champ d'image (optique), etc.

Transfert : transfert de chaleur (technique thermique), transfert de charge (électronique, nucléaire), transfert de masse (chimie, physique nucléaire), transfert de neutrons (nucléaire), transfert de porteur de charge (électronique), transfert de quantité de mouvement (mécanique), transfert d'impulsion (mécanique), transfert radiatif (radiations).

Les termes techniques complexes reflètent les progrès enregistrés et les réalités de plus en plus complexes du domaine de la technique : moteur à induction à rotor bobiné, moteur à condensateur à deux capacités, moteur automobile deux temps trois cylindres, module d'élasticité en traction, signal électrique modulé en largeur d'impulsion, coefficient de contraction par torsion, résistance à la torsion statique.

Pour ce qui est de la traduction des termes composés du français au roumain, on peut distinguer cinq situations :

1. Le terme en roumain est la traduction fidèle du terme français, ce qui suppose le même procédé de formation terminologique dans les deux langues:

Exemples : transfert de chaleur - transfer de căldură ; lumière anodique - lumină anodică ; masse active - masă activă ; mécanisme à cliquet - mecanism cu clichet.

2. Le transfert d'une langue à l'autre s'accompagne du changement de classe grammaticale d'un des éléments entrant dans la structure du terme composé français :

Exemples : mémoire à accès lent - memorie lentă au lieu de memorie cu acces lent ; marquage isotopique - marcare cu izotopi au lieu de marcare izotopică ; filament chauffant - filament de încălzire au lieu de filament încălzitor;

3. Le terme en roumain contient un élément qui ne se trouve pas en correspondance directe avec un élément du terme français entrant dans la composition du syntagme terminologique. Une documentation insuffisante de la part du traducteur mènerait à des calques fautifs.

Exemples : " chapeaux de bielle " où le mot " chapeaux " n'a pas été traduit par son équivalent pălării mais par capace (" couvercles ") ; " constante d'amortissement " trouve son correspondant en coeficient de amortizare (coeficient = coefficient) et non pas constantă de amortizare ; "domaine cathodique " est rendu par spaţiu catodic (spaţiu = espace) et non pas domeniu catodic ; " nombre de charge " sera traduit par număr atomic (atomic = atomique) et non pas număr de sarcină.

Il est à noter que les termes techniques tels que " tête de bobine ", " tête d'articulation ", " tête de câble ", "gorge de rupture ", " gorge de filetage ", " œill de poisson ", " pied monté ", " pied à coulisse ", " bras pivotant " sont des métaphores anthropomorphiques, nées par analogie entre les parties du corps humain et les dispositifs ou les objets qu'elles sont appelées à 
désigner. C'est ce que R. Kocourek(19) appelle le recours à « l'emploi figuré dans la formation terminologique ". Ce va-et-vient entre le lexique usuel et les lexiques spécialisés qu'Al. Cuniţă(20) appelle " frange d'interférence " peut constituer un obstacle pour le traducteur du texte technique, car le sens du mot commun change selon le domaine où il est utilisé : à titre d'exemple, le terme " tête de bobine " a comme correspondant cap de bobină (cap signifiant "tête ") alors que " tête de voûte " sera rendu par faţa arcului / faţa bolţii (faţa signifiant " face ").

4. Le terme roumain est obtenu à l'aide d'une traduction par paraphrase du terme français :

Exemples : atome engendré/atome fils - atom rezultat din dezintegrare; plateau de fonte - disc de rodat din fontă ; plateau monte et baisse - masă cu mecanism de ridicat ; tête bouterollée - cap semirotund format cu buterolă.

5. Tous les constituants du terme français se trouvent en correspondance indirecte avec les constituants du terme roumain équivalent :

Exemples : plateau suiveur - disc mobil de presare ; tube compteur à décades - decatron; tube d'arrivée d'essence - conductă de combustibil ; jupe de conditionnement d'air - camătă de aer.

\section{Conclusions}

Loin d'être exhaustive, cette étude nous a permis de présenter quelques problèmes que la traduction technique du français au roumain peut soulever au niveau lexical et sémantique. Nous nous sommes arrêtées à des situations qui peuvent constituer des sources d'ambiguités ou de faux sens : emploi figuré dans la formation terminologique, termes formés à partir d'opérateurs suffixaux ambivalents, termes polysémiques dans la langue de départ, calques fautifs. Il est à souligner, une fois de plus, le rôle déterminant de l'étape de documentation et du choix terminologique dans le processus de la traduction spécialisée. Une compétence linguistique limitée à la connaissance des règles grammaticales ne peut pas assurer la réussite d'une bonne traduction. Le traducteur se doit de mener une activité intense de documentation afin de comprendre et de faire comprendre l'information qu'il doit rendre dans la langue d'arrivée. Le choix des termes précis mènera à une bonne compréhension du texte par son destinataire final. Enfin, le recours à un spécialiste du domaine garantit la réussite d'une traduction spécialisée.

ana_marina_hotmail.com

ec_ilinca@yahoo.com

(19) $1982: 146$.

(20) $1982: 146$ 


\section{Bibliographie}

BALAN-MIHAILOVICI, Aurelia, 2005. " Neologia şi structura neonimelor » in Studii şi cercetări lingvistice, LVI, 1-2, p. 23-29.

BEAUNE, Jean-Claude, 1988. " La vulgarisation scientifique : l'ombre des techniques » in JACOBI, Daniel, SCHIELE, Bernard, (dir.), Vulgariser la science, le procès de l'ignorance, Paris, Champ Vallon, diffusion PUF, Paris.

BELL, T. Roger, 1995. Translation and translating: theory and practice, Londres, Longman.

COTEANU, Ion, SALA, Marius, 1987. Etimologia şi limba românã. Principii-probleme, Bucarest, Ed. Acad. Republicii Socialiste România.

CRISTEA, Teodora, 2007. Stratégies de la traduction, Ille édition, Bucarest, Editura Fundaţiei România de Mâine.

CUNIŢĂ, Alexandra, 1980. "La structure lexicale ", in MICLAU, Paul (éd.), Introduction à l'étude des langues de spécialité, Presses Universitaires de Bucarest.

FROELIGER, Nicolas, 2003. "Binaire et liminaire : la forme en traduction technique ", in Revue française de linguistique appliquée 2/2003 (Vol. VIII), p. 33-42.

GENTILHOMME, Yves, 1984. "Les faces cachées du discours scientifique, réponse à Jean Peytard ", in PEYTARD, Jean, JACOBI, Daniel, PETROFF, André, Langue française : Français technique et scientifique - reformulation, enseignement, 64/1984, Paris, Larousse, p. 28-37.

JACOBI, Daniel, 1984. "Du discours scientifique, de sa reformulation et quelques usages sociaux de la science ", in PEYTARD, Jean, JACOBI, Daniel, PETROFF, André, Langue française : Français technique et scientifique - reformulation, enseignement, 64/1984, Paris, Larousse, p. 37-52.

GILE, Daniel, 2004. La traduction. La comprendre, l'apprendre, Paris, Presses Universitaires de France.

Grand dictionnaire encyclopédique Larousse, 1985, Paris, Larousse, 10 vol.

GUILBERT, Louis, 1973. "La spécificité du terme scientifique et technique » in Français moderne, 17, 5-17.

KOCOUREK, Rostislav, 1982. La langue française de la technique et de la science : vers une linguistique de la langue savante, 2e éd. augmentée, refondue et mise à jour. Wiesbaden, Oscar Brandstetter Verlag.

LERAT, Pierre,1993. Les langues spécialisées, Paris, PUF.

LERAT, Pierre, 1997. " Approches linguistiques des langues spécialisées ", in ASp [En ligne], 15-18, mis en ligne le 16 avril 2012, consulté le 26 septembre 2012.

URL : http://asp.revues.org/2926 ; DOl : 10.4000/asp.2926. 
MACREA, Dimitrie, 1970. Terminologia ştiinţifică şi tehnică în limba română contemporană, dans Studii de lingvistică română, EDP, Bucureşti, p. 29-37.

MORTUREUX, Marie-Françoise, 1995. "Les vocabulaires scientifiques et techniques ", in Les Carnets du Cediscor [En ligne], 3 | 1995, mis en ligne le 25 septembre 2009, consulté le 08 octobre 2012. URL : http://cediscor.revues.org/463.

PETIT, Gérard, 2001. "L'introuvable identité du terme technique ", in Revue française de linguistique appliquée VI, 2, p. 63-79.

REGA, Lorenza, 2001. La traduzione litteraria. Aspetti e problemi, Turin, UTET.

SCARPA, Frederica, 2010. La Traduction spécialisée : La médiation linguistique et les langues de spécialité, University of Ottawa Press.

ȚITEICA, Radu, FIUCIUC, Natalia, 2002. Dicţionar de ştiintă şi technică francez-roman, Bucarest, Editions Niculescu.

VIGNER, Gérard, MARTIN, Alix, 1976. Le français technique, Paris, Hachette coll. "Le français dans le monde".

Elena-Cristina llinca est chargée de cours titulaire au Département des Langues Étrangères Appliquées, Faculté des Lettres, Université de Piteşti, Roumanie. Elle enseigne la théorie et la pratique de la traduction, le management de l'activité du traducteur et des travaux dirigés (traductions technico-scientifiques, correspondance commerciale). Ses derniers travaux portent sur la traduction spécialisée, notamment la traduction technico-scientifique. Elle travaille comme traductrice indépendante depuis 2005.

Ana-Marina Tomescu est maitre de conférences au Département des Langues Étrangères Appliquées, Faculté des Lettres, Université de Piteşti, Roumanie. Principales disciplines enseignées : Langages de spécialité, Phraséologie, Cours pratique - langue française : traductions économiques, grammaire. Travaux dirigés - langue française pour les domaines de l'économie, de l'administration publique et du droit. Ses dernières recherches portent sur la traduction spécialisée et les langues de spécialité. 